\title{
MECHANICAL ANALYSIS OF A PIEZOELECTRIC SENSOR USED FOR DAMAGE DETECTION IN A STEEL STRUCTURE JOINT
}

\author{
NOBUHIRO SHIMOI, CARLOS CUADRA \& HIDETO KANNO \\ Faculty of System Science and Technology, Akita Prefectural University, Japan
}

\begin{abstract}
Recently, sensors that use piezoelectric materials are studied to detect impacts, number of impact occurrences, vibrations, etc. in structures. One of them uses a piezoelectric film attached to a thin steel plate that is installed on a steel column-beam joint to investigate its mechanical behaviour. Since the piezoelectric material reacts when sudden changes occur, the piezoelectric film is covered by a thin glass plate, and it is expected that when glass plate cracks due to an applied load stress, the film will react by emitting a voltage signal. In this way it might be possible to detect certain level of damage in a structural steel joint. Using this sensor system, static tests of steel beam-column joints were carried out and the applicability of the sensor was verified. However, the sensor reaction begins at high levels of deformation and the cracking of the glass does not occurs in the central part of the sensor plate as it was expected. Then is this research a structural analysis of the sensor steel plate is performed to analyse the stress distribution and the possible causes of glass failure at the ends of the sensor plate. The load test was found to produce a bending moment on the sensor plate and this moment has a great influence on the location of the glass crack. Also, for reverse loading, the steel plate is subject to compressive stresses and buckling failure must be considered.
\end{abstract}

Keywords: piezoelectric sensor, seismic damages, compression failure, steel structure failure.

\section{INTRODUCTION}

During the 1995 Kobe earthquake, many steel structures were damaged due to large lateral displacements caused by input motions. Assessing the level of damage is an important task in estimating the security and safety of a building after an earthquake. If the number of buildings to be evaluated is very large, it is desirable to have an indicator of the level of stress or displacement experienced. This indicator can be obtained if appropriate sensors are installed in the building.

In steel frame buildings, generally the connections are bolted or welded joints. In the case of bolt fixing, when a dynamic external force such as impact, vibration, or thermal load (expansion) acts on the bolted joint, the bolt often loses its fixing force due to loosening of the nut and therefore, its fixing function is lost. In the case of welded joints, there are few accidents due to vibrations and loosening of the nuts, however, due to the thermal effects during welding, brittleness occurs around joints.

Inspection methods for welded joints include non-destructive inspection techniques and visual inspection, including x-ray analysis techniques, image analysis techniques using ultrasonic waves and magnetism, and hammering. Visual inspection techniques are also effective tools.

Using the finite element method, it is difficult to realistically express crack propagation and fracture analysis even in the case of the initial state of the weld. Therefore, it is also difficult to model the aging effects of a welded joint. In Japan, which has experienced the Great East Japan Earthquake, when a large earthquake occurs with a seismic intensity of six or more, according to the architectural design standards the entire building is plasticized without collapse of the building, absorbing the seismic energy and saving human lives. In other developed countries that have also experienced major earthquakes, there is no 
monitoring report of long-term robustness using precise measurement focusing only on joints.

In this research a piezoelectric sensor is studied for the detection of damage. The sensor consists of a piezoelectric film mounted on a thin steel plate and covered with a small, thin glass plate. The idea is that when a large deformation occurs, the glass plate breaks, at which point the piezoelectric material emits a voltage signal that is indicative of a certain level of damage. To investigate the stress distribution that affects the sensor response, a structural analysis of the sensor's steel plate is performed. This analysis helps explain the reasons for the location of the glass failure at the ends of the sensor plate. From the analysis it was found that the load test produces a bending moment on the sensor plate and this moment has a great influence on the location of the glass crack. For reverse loading, the steel plate is subjected to a compressive stress and the stress distribution is also affected by buckling.

\section{EXPERIMENTAL TESTS}

A piezoelectric sensor developed to detect the level of damage in the joint of steel structures was experimentally studied through a series of static test. These tests consisted of full-scale test on beam-column joint steel specimen and automated tests using a robotic system.

\subsection{Sensor characteristics}

In this research, the developed sensor consists of a piezoelectric film that is placed on a steel plate and covered with a layer of glass, as shown in Fig. 1. A special glue is used to fix the glass. This glue is a silicone that requires the application of ultraviolet rays to harden.

The steel base or plate of the sensor allows the detection system to be installed in a structural joint as can be seen in Fig. 2.

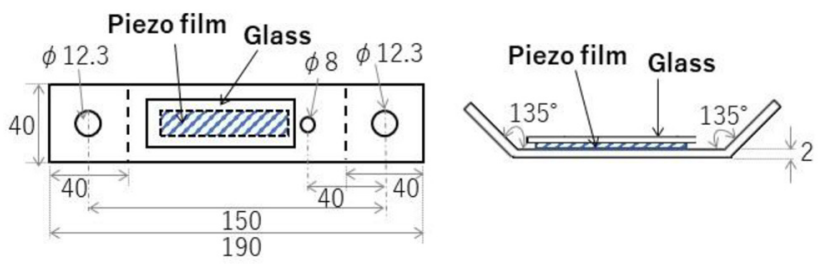

Figure 1: Piezoelectric sensor systems.

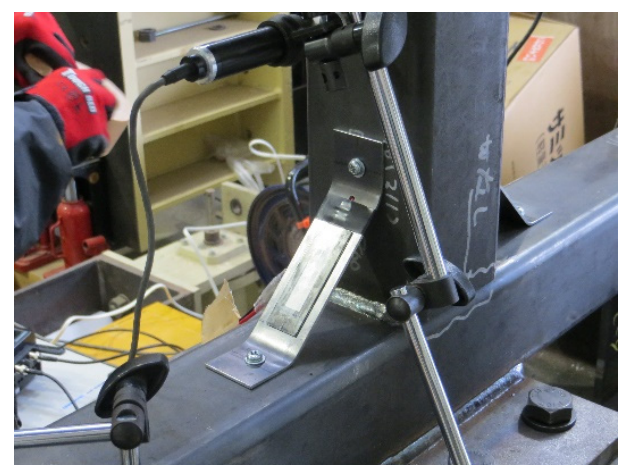

Figure 2: Sensor systems installed at beam-column joint. 
The proposed piezoelectric sensors use piezoelectric films. This film could be, for example, DT2-028K film $(16 \mathrm{~mm} \times 73 \mathrm{~mm})$ and LDT1-028K film $(16 \mathrm{~mm} \times 73 \mathrm{~mm})$ shown in Fig. 3. These are commercial multipurpose piezoelectric sensors to detect physical phenomena such as vibrations or impacts. The piezoelectric film element is laminated to a polyester sheet and produces a usable electrical signal output when forces are applied to the sensing area. The dual wire lead attached to the sensor allows easy connection to a monitoring circuit or device to process the signal.

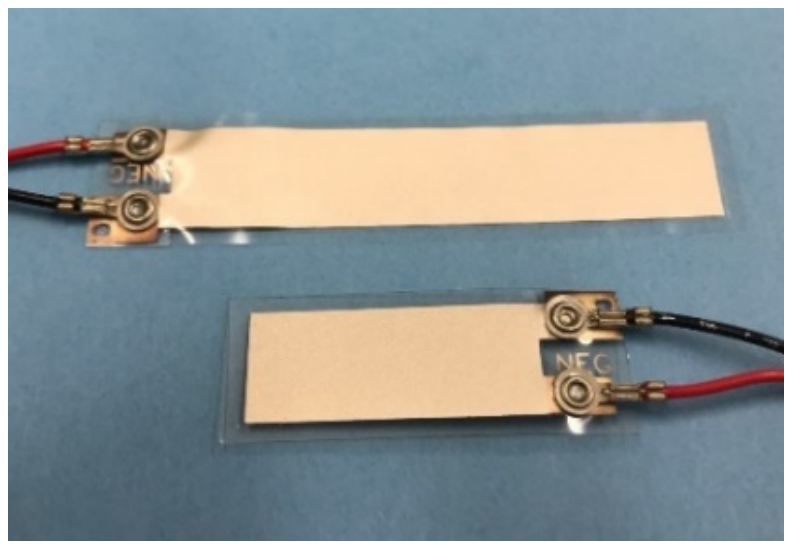

Figure 3: Piezoelectric films.

\subsection{Static test on full scale specimen}

The applicability of the proposed sensor was investigated by lateral loading experimental tests on full-scale specimens. The cross section of steel elements (beam and column) is a $100 \mathrm{~mm} \times 100 \mathrm{~mm}$ box section with a $6 \mathrm{~mm}$ thin. This steel section is specified by the Japanese Industrial Standards as carbon steel square-tube for general structure (STKR400). The column and beam elements are connected perpendicularly using a fillet weld. The resulting test specimen has an inverted T shape as shown in Fig. 4.
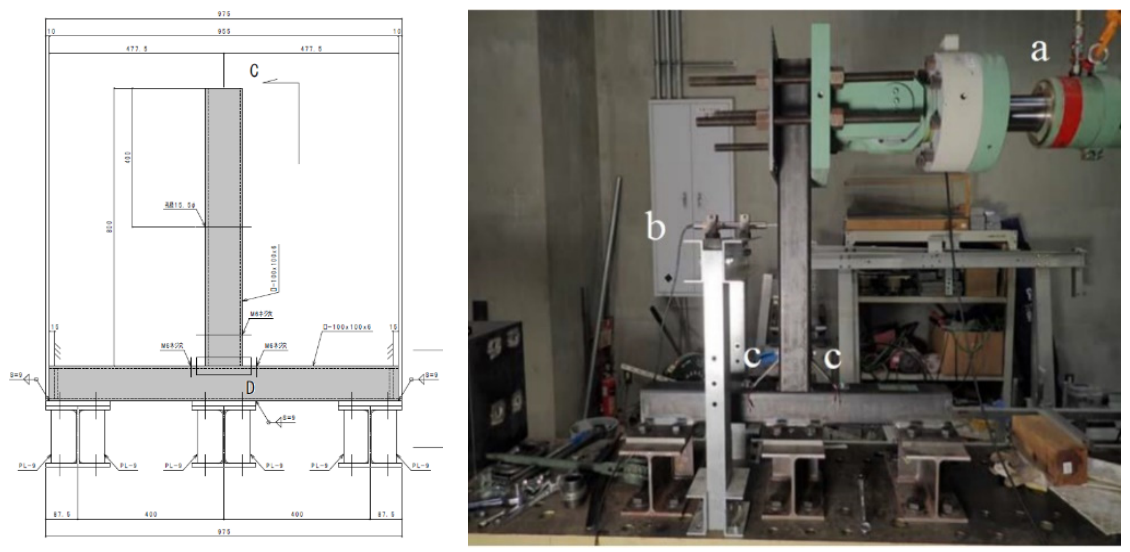

Figure 4: Test specimen and full-scale static test setup. 
The horizontal part of the specimen is attached to a reaction system while the lateral force is applied to the free end. Piezoelectric sensors were installed at both corners of the specimen connecting the beam and column via the small diagonal steel plate that contains the piezoelectric sensor. Then, when lateral force is applied to the specimen it is assumed that this plate works in tension or compression depending on its position. Free end lateral displacement and lateral load were measured using displacements transducers and load cell, respectively.

Monotonic lateral load was applied reaching a maximum load of the order of $16 \mathrm{kN}$ and the maximum displacement was $35 \mathrm{~mm}$ which corresponds to a drift angle of $1 / 20$. Results for load history and sensor response are show in Fig. 5. Piezoelectric sensors show a first response at a drift angle of the order of 1/100 (Crack 1), and a second response is clearly observed at a drift angle of 1/50 (Crack 2). The drift angles at which piezoelectric sensors respond are relatively large. Therefore, it is necessary to improve the setup of sensors to obtain responses at early steps of loading. Although these preliminary results permit to verify the response of sensors for large deformation it can be concluded that it is possible to estimate the level of deformations using these simple piezoelectric sensors.

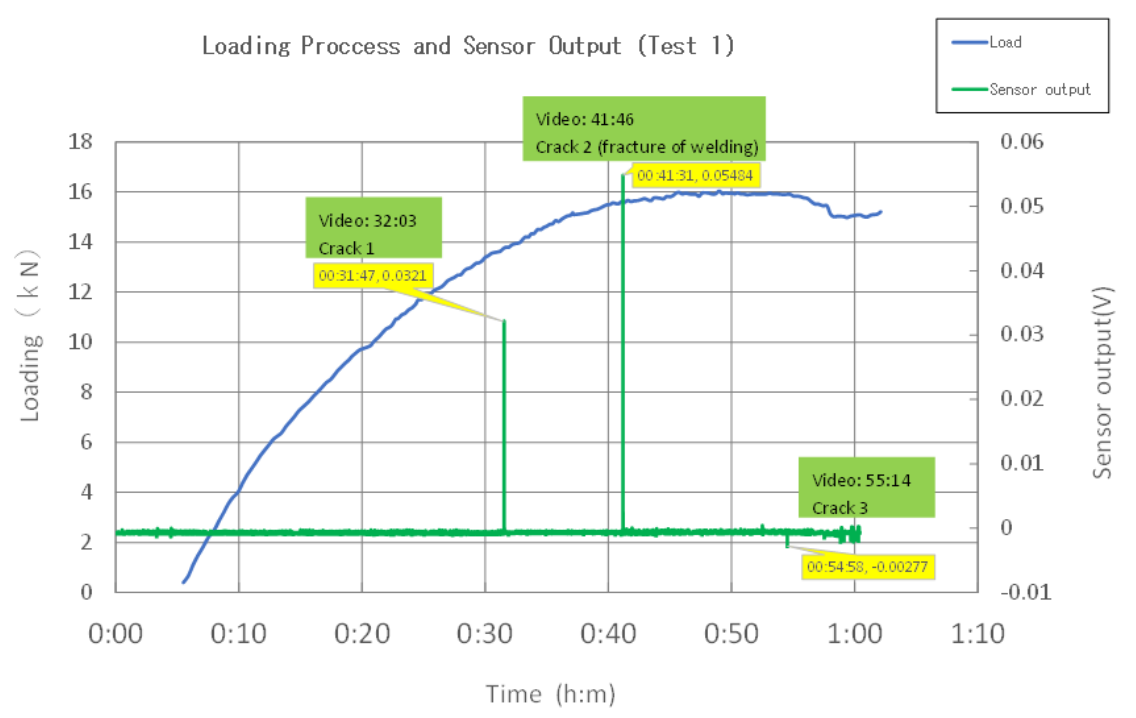

Figure 5: Test specimen and full-scale static test setup.

\subsection{Static test on systematic test device (robot SALLY)}

Full scale test to study the response of the piezoelectric sensor at the same time as the response of the structure is not an optimal procedure. Therefore, to study only the sensor response, an automated procedure was designed. This automated test allows studying the behaviour of the piezoelectric sensor system under different load conditions and allows a variation of the dimensions and shape of the sensor. The automated system shown in Fig. 6 is called as a Sally robot that consists of a horizontal beam and a vertical element connected by a pin joint with the horizontal element. The load is applied at the top of the vertical element by a screw system controlled from a computer and the load is measured by an electric load cell and the displacement stroke is measured by electrical transducers. For positive load as 
shown in Fig. 6, the sensor is supposed to work in tension and for negative load direction, the sensor should work in compression.
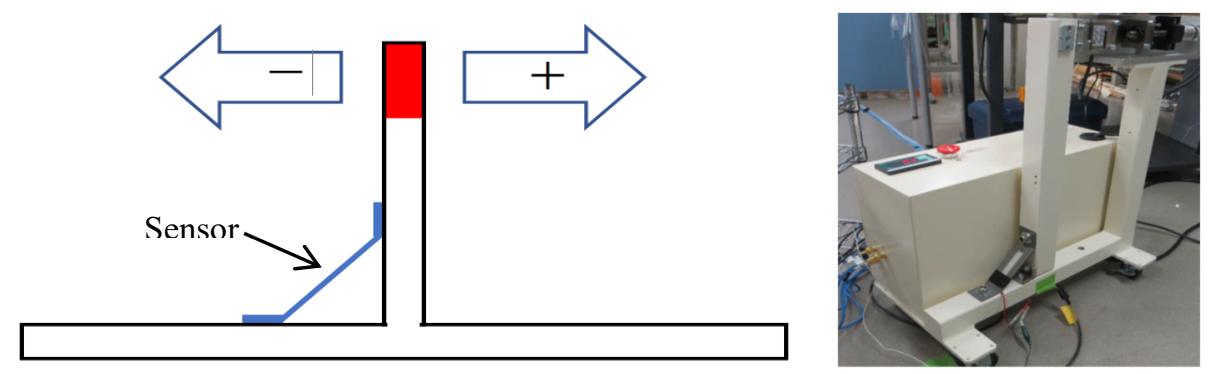

Figure 6: Automatised test system (robot Sally).

The results for loading in positive and negative direction are shown in Figs 7 and 8. The sensor response is plotted in red colour and the sensor reaction (or glass breakage) occurs at $8 \mathrm{~mm}$ and $10 \mathrm{~mm}$ for positive and negative directions respectively. This is a large level of displacement corresponding to a drift angle of $1 / 75$ to $1 / 50$, which are important values for structural deformations. Therefore, it is necessary to redesign the dimensions of the sensor system to obtain reactions to smaller deformations.
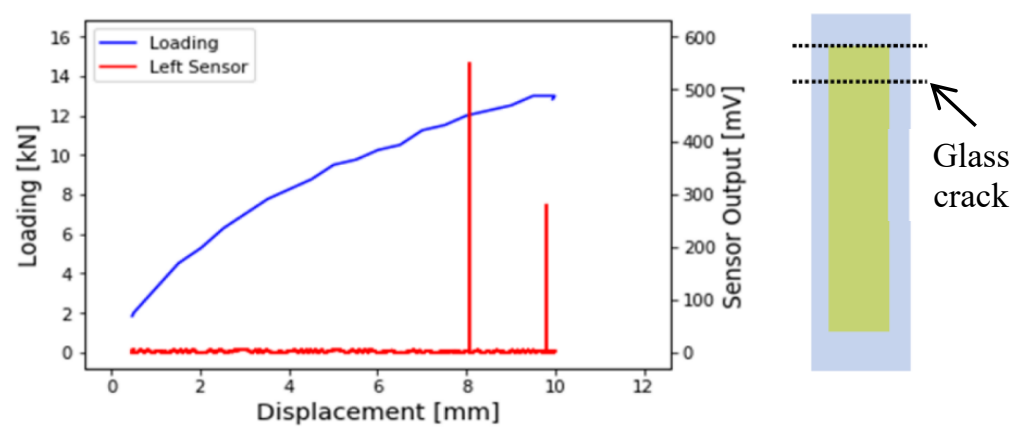

Figure 7: Results for loading in positive direction.
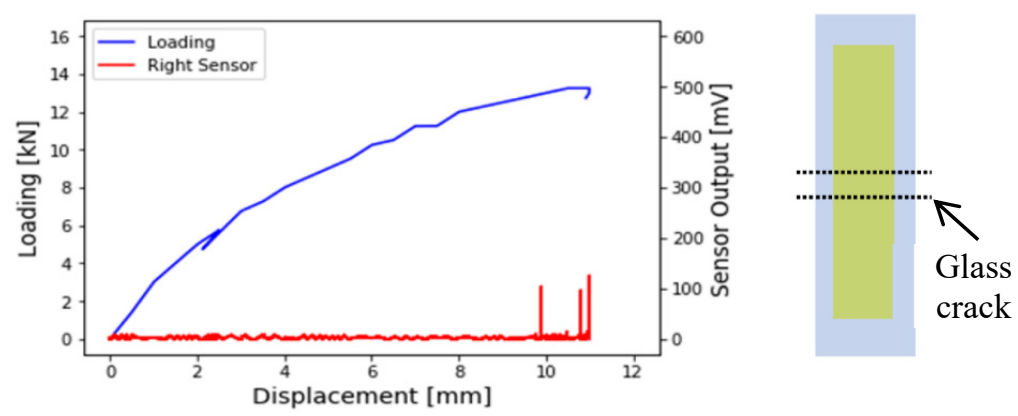

Figure 8: Results for loading in negative direction. 
On the right side of Figs 7 and 8, the location of the glass cracking is indicated by dotted lines. It can be observed that in case of positive load (the sensor plate is supposed works in tension) the cracks are located at the upper part of the sensor plate and not at the central part as originally planned. In the case of negative load, the sensor plate works mainly in compression and the cracks are located in the central part of the plate. To ensure that cracks always occur in central part of the plate, it is necessary to decrease the cross section in the central part to induce a stress concentration in this part.

\section{FEM ANALYSIS}

In case of automated tests, a finite element analysis is performed to investigate the stress distribution on the sensor plate due to both load patterns. The model of the sensor plate is shown in Fig. 9. Plane shell elements are use and it is also considered the hole that is used to pass the electric cables. The objective is also to investigate the effect of this hole in the stress distribution. Both ends of the plate are considered pinned joints. The sensor plate is fixed to the horizontal and vertical element by means of bolts and therefore it is considered a good approximation to model these boundaries as pinned joints. Then, deformation of the upper right part of the plate is applied as external action that causes the stress in the central plate.
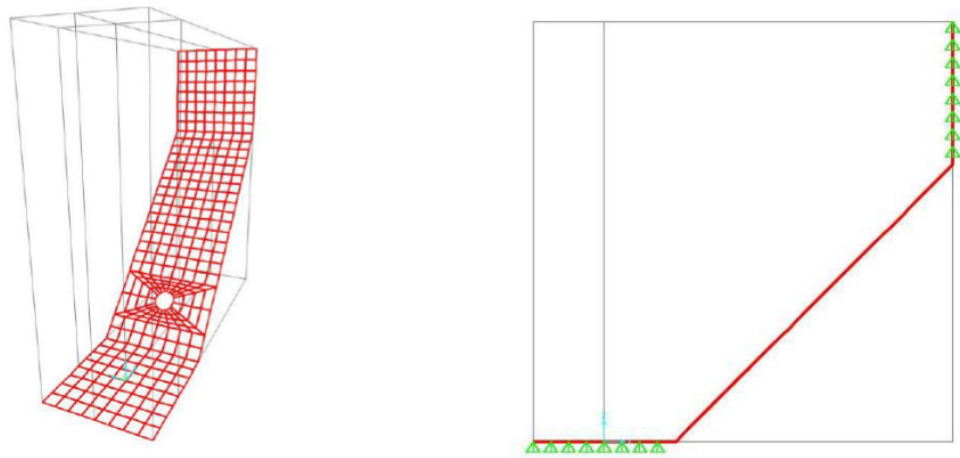

Figure 9: Finite element model of sensor plate.

The distribution of stress and deformation due to positive load is shown in Fig. 10. Since the displacement with linear distribution is applied in the upper right part of the plate, this part rotates as a rigid body. The deformation shows that flexion due to bending moment is concentrated at upper part of the sensor plate. Therefore, this moment causes the stresses on the plate. The concentration of this stress (which is tensile stress) causes the cracking of glass at the upper part of the sensor.

In case of negative load, compression stress is originated at upper part as is show in Fig. 11. However, in this case since a thin plate is subjected to compression stress, it is necessary to consider the effect of the buckling behaviour. The results considering the buckling effect are shown in Fig. 12 and it can be observed that the stresses are concentrated in the central part of the sensor. This stress is then believed to cause cracking of the glass in the central part of the plate.

From the analysis of both load cases, it was observed that the small hole in the plate does not significantly affect the stress distribution. 

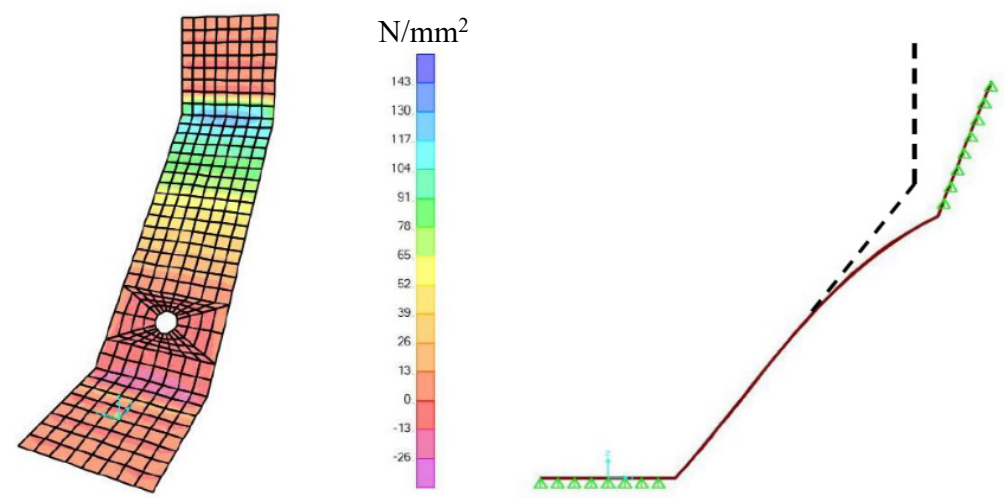

Figure 10: Results for loading in positive direction.
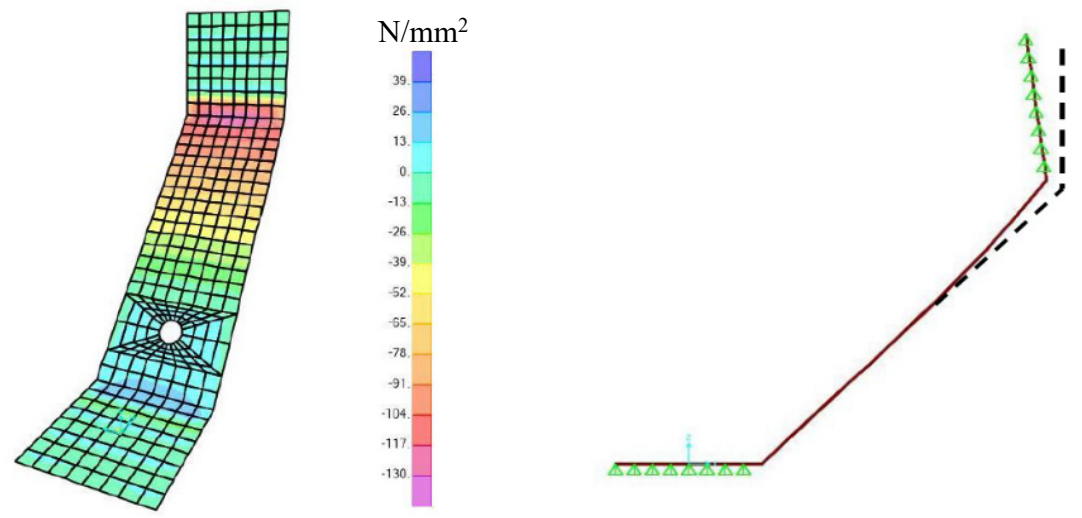

Figure 11: Results for loading in negative direction.
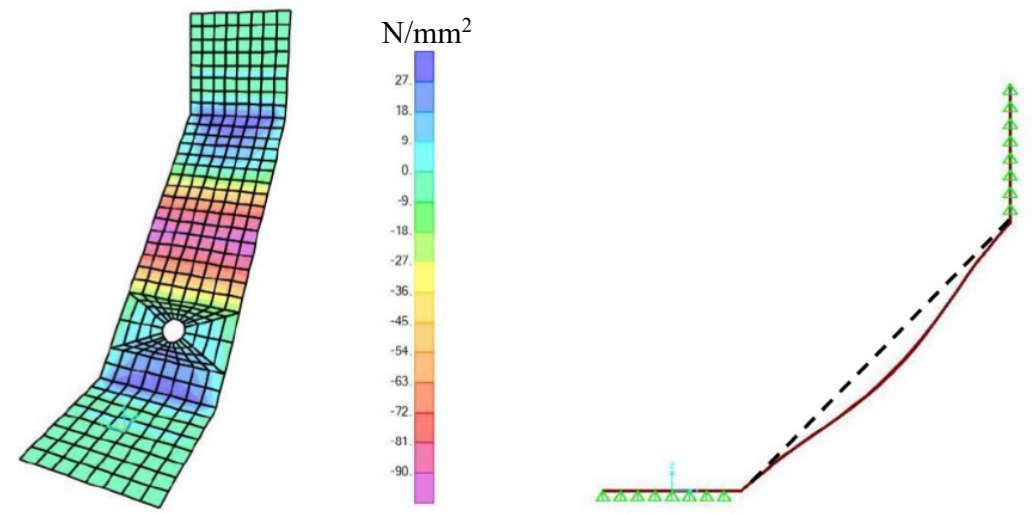

Figure 12: Results for loading in negative direction and considering buckling effect. 


\section{CONCLUSIONS}

In this research, analysis of a new piezoelectric sensor system was performed to identify its behaviour pattern. The sensing system consists of a thin rectangular piezoelectric film mounted on a steel base plate and covered by a piece of glass. Cracking of the glass during loading allows a reaction of the piezoelectric material and this reaction indicates some level of damages of a structure. The applicability of this piezoelectric sensor system to detect the occurrence of failure was experimentally verified using a steel specimen subjected to lateral loading. Sensor reaction was also investigated using an automated robotic test setup.

Although preliminary results allow to verify the response of sensors for large deformations, it can be concluded that it is possible to estimate the level of deformations using this simple piezoelectric sensor.

The finite element analysis method was used to investigate the stress distribution affecting the sensor reaction and the location of the cracks in the part of the sensing glass. It was found that the steel base plate works in bending and the stresses originate by bending moment. In the case of compressive stresses, it was shown that it is important to consider the buckling effect.

For a regular and stable sensor response, it might be desirable to have the stress concentration in the middle of the sensor plate. This concentration could be achieved by changing the cross section of the plate near the centre.

\section{ACKNOWLEDGEMENT}

The authors acknowledge Japan Society for the Promotion of Science for the Grants-in-Aid for Simple Autonomous Health Monitoring System for Infrastructure Using Piezoelectric Limit Sensors, under which this research was conducted.

\section{REFERENCES}

[1] Cuadra, C., Shimoi, N., Sasaki, T. \& Taguchi, T., Preliminary evaluation of piezoelectric sensors for the prediction of compression failure of brick masonry components. WIT Transactions on The Built Environment, vol. 153, WIT Press: Southampton and Boston, 2015. DOI 10.2495/STR150501.

[2] Shimoi, N., Nishida, T., Obata, A., Nakasho, K., Madokoro, H. \& Cuadra, C., Comparison of displacement measurements in exposed type column base using piezoelectric dynamic sensors and static sensors. American Journal of Remote Sensing 4(5), pp. 23-32, 2016.

[3] Cuadra, C., Shimoi, N. \& Saijo, M., Dynamic characteristics of a bridge estimated with new bolt-type sensor, ambient vibration measurements and finite element analysis. International Journal of Safety and Security Engineering, 6(1), pp. 40-52, 2016. 